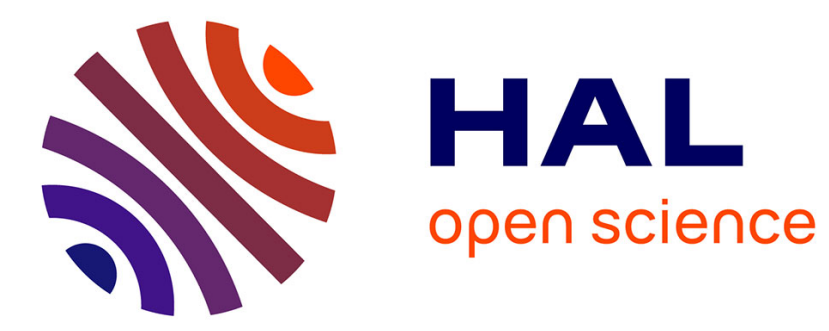

\title{
Improving arterial spin labeling by temporal filtering
}

Jan Petr, Jean-Christophe Ferré, Jean-Yves Gauvrit, Christian Barillot

\section{To cite this version:}

Jan Petr, Jean-Christophe Ferré, Jean-Yves Gauvrit, Christian Barillot. Improving arterial spin labeling by temporal filtering. SPIE Medical Imaging 2010: Image Processing, Feb 2010, San Diego, United States. pp.n.a, 10.1117/12.843960 . inserm-00601146

\section{HAL Id: inserm-00601146 https://www.hal.inserm.fr/inserm-00601146}

Submitted on 16 Jun 2011

HAL is a multi-disciplinary open access archive for the deposit and dissemination of scientific research documents, whether they are published or not. The documents may come from teaching and research institutions in France or abroad, or from public or private research centers.
L'archive ouverte pluridisciplinaire HAL, est destinée au dépôt et à la diffusion de documents scientifiques de niveau recherche, publiés ou non, émanant des établissements d'enseignement et de recherche français ou étrangers, des laboratoires publics ou privés. 


\title{
Improving arterial spin labeling data by temporal filtering
}

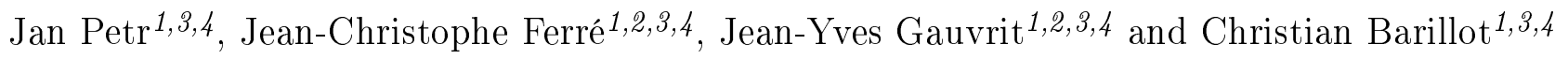 \\ ${ }^{1}$ INRIA, VisAGeS Project-Team, F-35042 Rennes, France \\ ${ }^{2} \mathrm{CHU}$, University Hospital of Rennes, Radiology Dept., F-35043 Rennes, France \\ ${ }^{3}$ University of Rennes I, CNRS, UMR 6074, IRISA, F-35042 Rennes, France \\ 4INSERM, U746, F-35042 Rennes, France
}

\begin{abstract}
Arterial spin labeling (ASL) is an MRI method for imaging brain perfusion by magnetically labeling blood in brain feeding arteries. The perfusion is obtained from the difference between images with and without prior labeling. Image noise is one of the main problems of ASL as the difference is around $0.5-2 \%$ of the image magnitude. Usually, 20-40 pairs of images need to be acquired and averaged to reach a satisfactory quality. The images are acquired shortly after the labeling to allow the labeled blood to reach the imaged slice. A sequence of images with multiple delays is more suitable for quantification of the cerebral blood flow as it gives more information about the blood arrival and relaxation. Although the quantification methods are sensitive to noise, no filtering or only Gaussian filtering is used to denoise the data in the temporal domain prior to quantification. In this article, we propose an efficient way to use the redundancy of information in the time sequence of each pixel to suppress noise. For this purpose, the vectorial NL-means method is adapted to work in the temporal domain. The proposed method is tested on simulated and real 3T MRI data. We demonstrate a clear improvement of the image quality as well as a better performance compared to Gaussian and normal spatial NL-means filtering.
\end{abstract}

\section{INTRODUCTION}

Arterial spin labeling (ASL) is an MRI method for imaging brain perfusion by magnetically labeling blood in brain feeding arteries. The main advantage of ASL is the absence of contrast agents or ionizing radiation. This complete noninvasiveness makes it especially suitable for healthy patients and for repetitive follow-ups. ${ }^{1}$

There are two basic labeling schemes used for ASL - pulsed and continuous. They both function on a similar principle and differ in the design of acquisition sequence. In this article, we focus on pulsed ASL (PASL). However, the proposed filtering method can be as well applied on continuous ASL (CASL).

In PASL, an $180^{\circ} \mathrm{RF}$ pulse is applied before the acquisition to a thick slab inferior to the imaged slice to magnetically label the arterial blood. The labeled blood then continuous its movement in the vascular system, it reaches the slice of interest and perfuses the brain tissue. At a specified time after the labeling (inversion time - TIms) a standard EPI sequence is used to acquire a so-called labeled image. Such labeled image has slightly decreased intensity as a result of the presence of $180^{\circ}$ inverted spins from the labeled blood.

The same acquisition without prior blood labeling is repeated to obtain a control image. The difference image (control-labeled) reflects the magnetization of the inflowing blood in each voxel and it is directly proportional to the tissue perfusion. As the difference is usually around $0.5-2 \%$ of the control image magnitude, its signal-tonoise-ratio (SNR) is not sufficient for further analysis. Typically, 20-40 pairs of control and labeled images are acquired (for each slice and $T I$ ) and averaged to obtain SNR sufficient for cerebral blood flow (CBF) quantification or for visual analysis of the perfusion.

Several parameters need to be estimated to allow correct quantification of the CBF (tissue and blood relaxation times, equilibrium blood magnetization, arrival time of blood to tissue etc.). Values of most of these parameters were estimated for $1.5 \mathrm{~T}$ and $3 \mathrm{~T}$ scanners or they can be easily estimated for each individual from the standard ASL data. However, the arrival time $T A$ of the labeled blood varies in population, it is non-uniform across the brain ${ }^{2}$ and it cannot be extracted from an ASL image with a single $T I$ that is the common mode to study the brain perfusion. A special labeling sequence called QUIPSS ${ }^{3,4}$ was introduced to overcome this limitation. In QUIPSS, the labeled blood bolus is saturated at a certain time TS after the labeling and before the acquisition. The temporal length of the bolus is thus precisely defined to be TS. The inversion time is then chosen to be $T I>T S+T A$ for all pixels to ensure that the blood bolus has entirely passed through all pixels at 
the moment of acquisition. This renders the perfusion-weighted images less dependent on the arrival time and allows the correct quantification of CBF without the precise knowledge of $T A$.

This approach however does not work for the case of too long arrival times (for example patients with atherosclerosis). The solution in this case is to obtain more information about the inflow of blood by acquiring images for several different TIs. CBF can then be estimated using deconvolution ${ }^{5}$ or by fitting a perfusion model $^{6-8}$ to the data. Since all of these methods are sensitive to noise, a reliable denoising method can be of great importance for increasing quantification precision as currently only Wiener ${ }^{9}$ or Gaussian filtering ${ }^{10}$ is $^{\text {used }}$ for this purpose.

We propose a model-free method that uses redundancy of information in the time domain to denoise the multi-TI PASL sequence. The method searches for pixels with similar perfusion properties whose measured values differ only in noise. Averaging the intensities of those similar pixels then results in noise suppression. For this purpose, the NL-Means method ${ }^{11-13}$ is adapted to use the time neighborhood of each pixel instead of the spatial neighborhood. The performance of the method is validated first on a simulated dataset created by fitting a perfusion model ${ }^{8}$ to data from a healthy volunteer and also on a dataset obtained using more 140 pairs of control-labeled images instead of the usual 30 pairs. The proposed method is tested for different levels of noise and improved SNR is demonstrated.

\section{METHODS}

\subsection{The NL-means algorithm}

The NL-Means algorithm was first introduced by Buades et al. ${ }^{11}$ and it was successfully applied in the field of medical imaging. ${ }^{12}$ Contrary to the principle of most denoising methods which average intensities of voxels in the spatial neighborhood, NL-means reconstructs the value of each pixel by weighted averaging intensities of similar pixels that can be located anywhere in the image and not necessarily in the neighborhood. The weights reflect the similarity between pixels and are estimated by comparing the intensities in their neighborhoods.

Each filtered value $N L_{v}(i)$ is thus a weighted average of intensities of all pixels $v(j)$ from the noisy image $I$

$$
N L_{v}(i)=\sum_{j \in I} w(i, j) v(j)
$$

where the weights $w$ quantify the similarity between the pixels $i$ and $j$ using their spatial neighborhood. The square Euclidean distance of the intensities with Gaussian weighting is employed:

$$
w(i, j)=\frac{1}{Z(i)} e^{-\frac{\sum_{k \in K} \mid v(i+k)-v(j+k) \|^{2}}{h^{2}}},
$$

where $K$ defines the spatial neighborhood, $Z(i)$ is a normalization constant such that $Z(i)=\sum_{j} w(i, j)$ and $h$ is a smoothing parameter.

\subsection{NL-means in time}

The shape of the arterial input function as well as the tissue relaxation times and perfusion differ across the brain. However, we assume that there are regions or individual pixels with similar or equal behavior. ${ }^{2}$ Their intensities measured by ASL thus differ only in noise, see Figure 1. To use this property for denoising, we extend the vectorial NL-means ${ }^{13}$ to include a time neighborhood. The filtered value $N L_{v}(i, t)$ on position $i$ in image $V$ and with $T I$ equal to $t$ is obtained as

$$
N L_{v}(i, t)=\sum_{j \in V} w(i, j, t) v(j, t)
$$

The weights $w$ are estimated using the following equation that quantifies the pixel similarity

$$
w(i, j, t)=\frac{1}{Z(i, t)} \exp \left[\left(-\sum_{t^{\prime}}\left|v\left(i, t^{\prime}\right)-v\left(j, t^{\prime}\right)\right|^{2}-\sum_{k \in K}|v(i+k, t)-v(j+k, t)|^{2}\right) /\left(h^{2}\right)\right],
$$




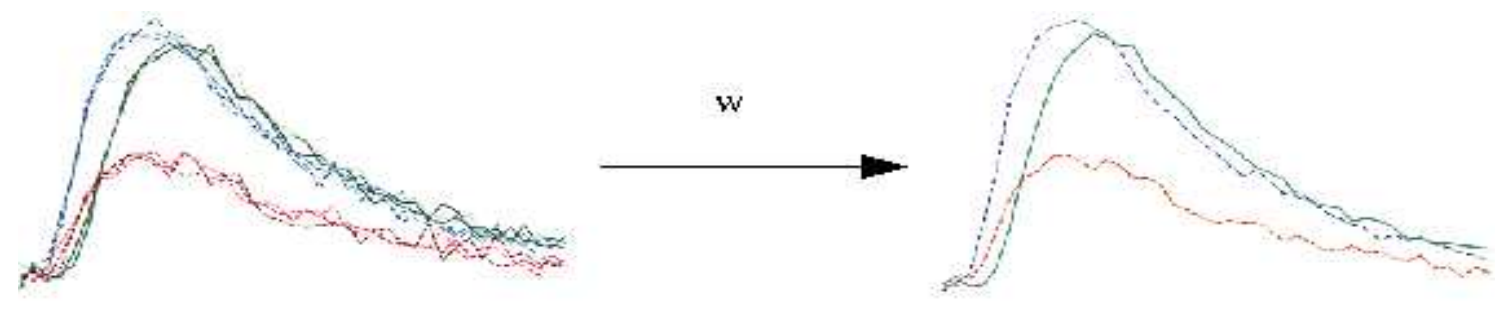

Figure 1. Illustration of the temporal NL-means filtering. Three different curves are on the left image - three samples of each curve with random noise are displayed. Averaging all nine curves together with appropriate weighting generates the result on the right with considerably lower level of noise.

where $Z(i, t)=\sum_{j} w(i, j, t)$. The smoothing parameter $h$ is set as proposed by Coupe et al. ${ }^{12}$

$$
h=2 \sigma^{2} N_{T},
$$

where $N_{T}$ is the number of $T I$ and $K$, and $\sigma$ is the standard deviation of noise.

The proposed filtering method assumes that the control-labeled image difference contains Gaussian noise. In theory, both the control and labeled ASL images contain Rician noise. Therefore, it may seem that the recently proposed Rician NL-means filtering ${ }^{14}$ would be more suitable for the filtering. However, the difference of controllabeled images is used as the input data. The difference is around $1 \%$ of the average image magnitude. The effect of Rician bias in the difference image is thus not so significant. Moreover, the parallel imaging is usually used in ASL. This can produce images with unequal distribution of noise variation. That makes the Rician NL-means filtering ${ }^{14}$ even less suitable as equal noise variation in all pixels is the key assumption of the method.

\subsection{Acquisition}

A healthy volunteer after giving a written informed consent was scanned on Philips Achieva 3T MRI scanner (Philips Medical Systems, Best, The Netherlands) with the following parameters: EPISTAR sequence with LookLocker strategy for sampling of multiple time-points, ${ }^{15} T R=4000 \mathrm{~ms}, T E=23 \mathrm{~ms}$, flip angle $=40^{\circ}$, matrix size $=$ $64 \times 64$, resolution $=3.6 \times 3.6 \times 6 \mathrm{~mm}$, SENSE factor $=2$, inter-slice gap $=0.6 \mathrm{~mm}, 150$ repetitions (control-labeled pairs) and cutoff velocity of crusher gradients $3 \mathrm{~cm} / \mathrm{s}^{16}{ }^{16}$ Four slices were acquired with TIs for the lowest slice $T I_{1} / \Delta T I / T I_{\text {last }}=200 \mathrm{~ms} / 200 \mathrm{~ms} / 3100 \mathrm{~ms}$ and an extra delay of $39 \mathrm{~ms}$ between slices. All the 150 repetitions were coregistered to compensate for the patient motion with the first one using $3 \mathrm{D}$ rigid-body transformation and a mutual information criterion.

The perfusion was estimated by fitting a perfusion model to the sequence of multi- $T I$ difference images (control minus labeled). ${ }^{8}$ The literature values of most of the model parameters relevant to the $3 \mathrm{~T}$ field were used: tissue relaxation time $T_{1}=1330 \mathrm{~ms}$, blood relaxation time $T_{b}=1664 \mathrm{~ms},{ }^{17}$ blood-tissue partition coefficient $\lambda=0.9 .{ }^{18}$ The blood flow, arrival time and bolus dispersion for each pixel were iteratively estimated by minimizing the squared difference between the model and the measured data in each pixel, see Figure 2.

\subsection{Dataset}

Three datasets were created to validate the proposed denoising method. The mean of the 150 repetitions was set as the ground truth of the first dataset. For each voxel and for each TI, the standard deviation over the 150 repetitions was estimated (assuming Gaussian distribution). The mean standard deviation $\sigma$ over all pixels and all TIs was computed. Gaussian noise with zero mean and standard deviation $\sqrt{N} \sigma$ was added to the ground truth image for $N$ from 1 to 30 to create 30 volumes with noise level similar to that of an average of 1 to 30 repetitions. These volumes were processed by the three methods described in Section 2 to assess their performance at different noise levels (the standard NL-means filtration with $3 \times 3$ neighborhood, the NL-means with the extra time-neighborhood of 5 pixels and a Gaussian filter with standard deviation 0.85 applied in the time domain $\left.{ }^{10}\right)$. The filtered images were compared with the ground truth image and the signal-to-noise ratio (SNR) was computed from the difference. 

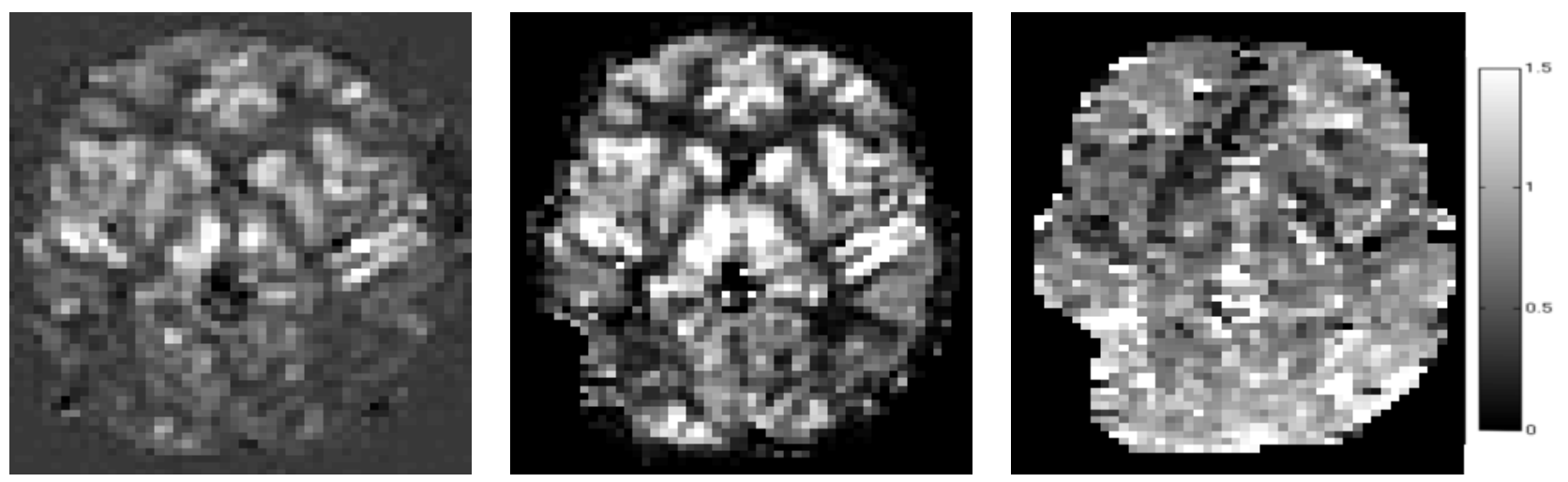

Figure 2. (left) The ASL image of the lowest slice for $T I=1200 \mathrm{~ms}$. (center) The cerebral blood flow as estimated using the Hrabe's model. (right) The estimated arrival time in seconds.

The estimated values of the Hrabe's perfusion model (Section 2.3) were used to generate the second dataset consisting of a sequence of volumes for TIs equal to those of the acquired data. The same validation process as for the first dataset was used.

Finally, the methods were executed on the dataset of 150 unprocessed repetitions using leave-one-out cross validation. A set of the first $N$ volumes out of the 150 was used as a noisy input and an average of the remaining $150-N$ volumes as the ground truth. The same was repeated with the second set of $N$ images (from the $N+1 s \mathrm{t}$ to the $2 N$ th image) etc. The average SNR of the $150 / N$ results was computed. The same process was repeated for $N=5,10,15,25,30$.

\section{RESULTS}

Relative performance of all the tested methods is nearly the same on all four slices as is documented on the case of simulated data, see Figure 3 right. Also the results are stable for all the images with different inversion times, see Figure 3 on the left. For this reason, the following results are displayed using mean SNR over all slices and inversion times. The overall signal is increasing for inversion times from $200 \mathrm{~ms}$ to around $1200 \mathrm{~ms}$ as the effect of the delayed arrival of the labeled blood. For higher inversion times, the signal is decreasing as a result of relaxation of the labeled spins and also as a result of repetitive excitation of the labeled spins in the imaged slice. Since the standard deviation of noise is constant for all slices and all inversion times, the SNR is changing with the overall signal.

The results on both simulated datasets show two times higher increase in SNR of the NL-means methods than in the Gaussian filtering, see Figure 4. The NL-means with time-neighborhood is approximately $1 \mathrm{~dB}$ better than the standard NL-means for all input images except for the first simulated dataset where the difference is negligible for more than 20 repetitions.

Qualitative comparison of the results on simulated datasets shows significantly less noise in the NL-means filtered result than in the simple Gaussian filtering, see Figure 5. The is no significant qualitative difference between the standard NL-means and the NL-means with the extended time-neighborhood.

The time NL-means offers $2 \mathrm{~dB}$ increase in SNR for the real data with 5, 10 and 15 repetitions and more than $0.5 \mathrm{~dB}$ for the images with more repetitions when compared with both other tested methods, see graph on Figure 6. The time NL-means results have qualitatively the highest level of noise suppression, while the structures are not oversmoothed, see 7.

\section{DISCUSSION AND CONCLUSIONS}

In this paper, we have proposed an extension of the NL-means filtering to the time domain. We have shown that, using this method, it is possible to denoise the ASL data without any prior knowledge or model. The method performance has been validated on a simulated data with added noise and also on a real dataset. Increased SNR 

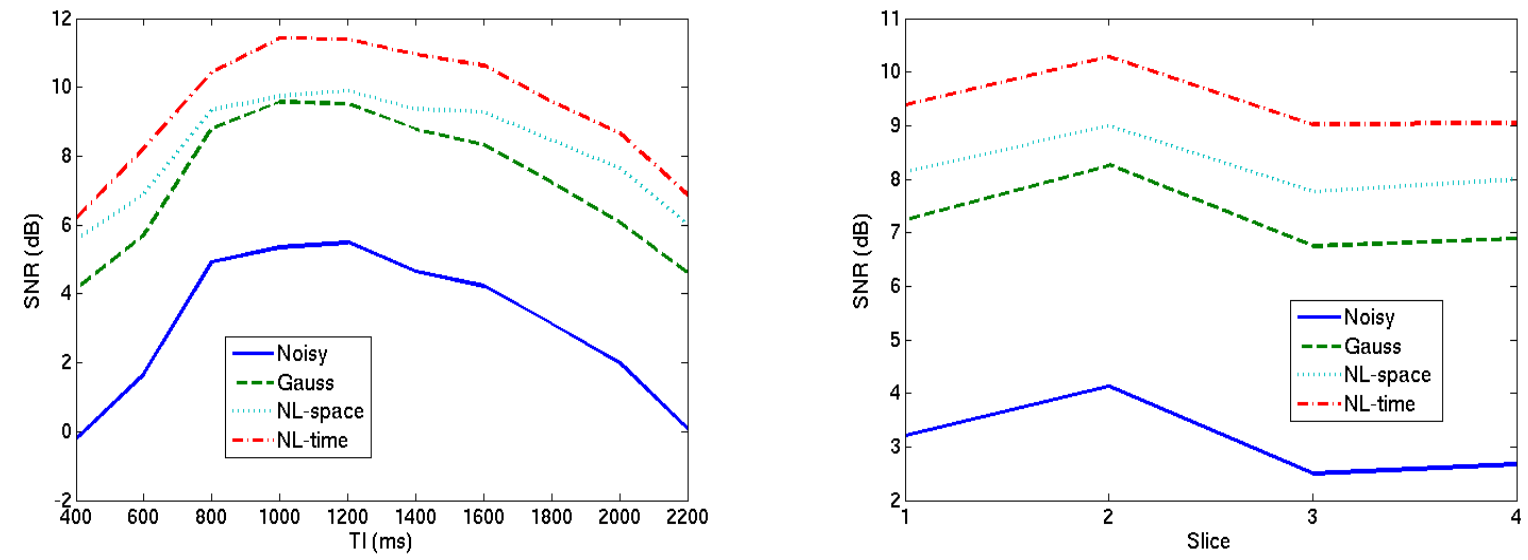

Figure 3. Result of filtering on the simulated dataset with added noise level corresponding to 20 repetitions. (left) The SNR averaged over all slices is shown for different TIs. (right) The SNR averaged over all TIs is shown for different slices.
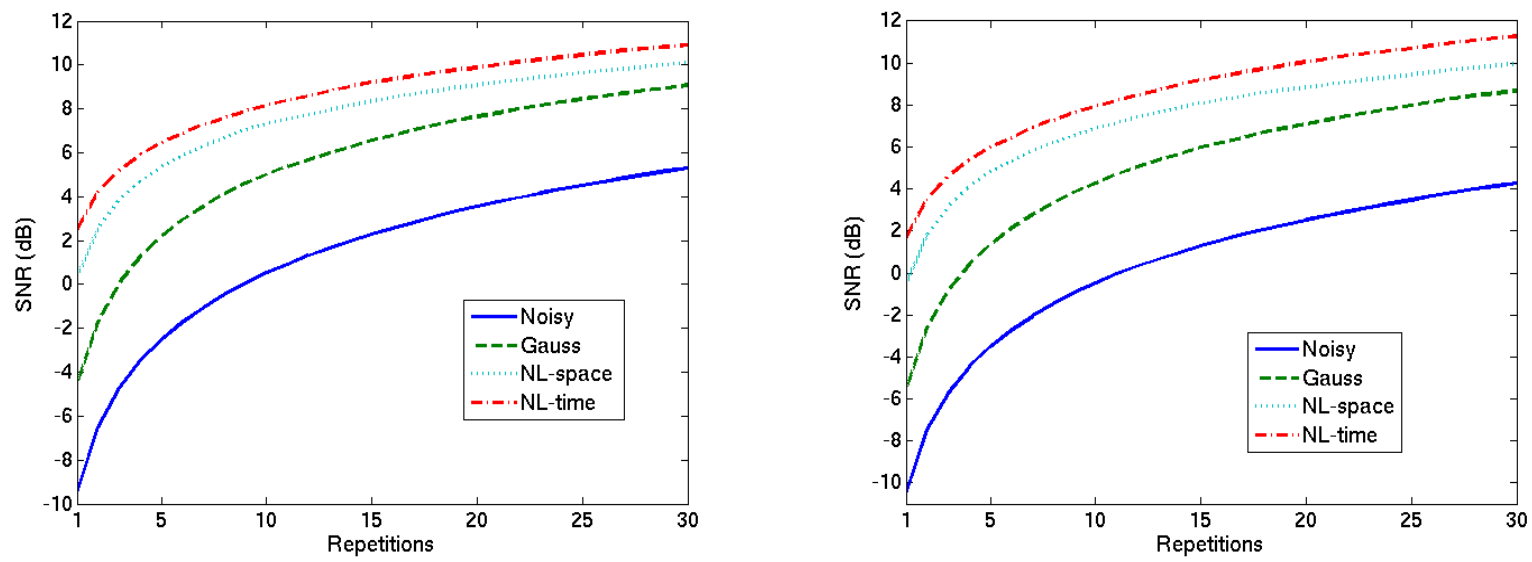

Figure 4. (left) First simulated dataset (noise added), (right) second simulated dataset (noise added to Hrabe's model). The mean SNR over all slices and inversion times is displayed for reconstructions of simulated images with noise level corresponding to $1-30$ repetitions. 


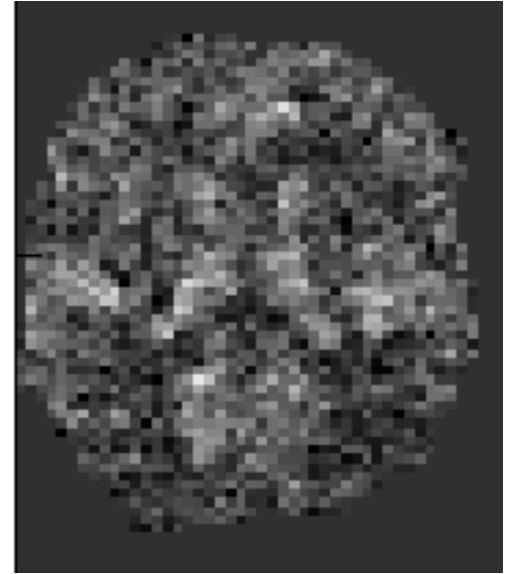

Noisy

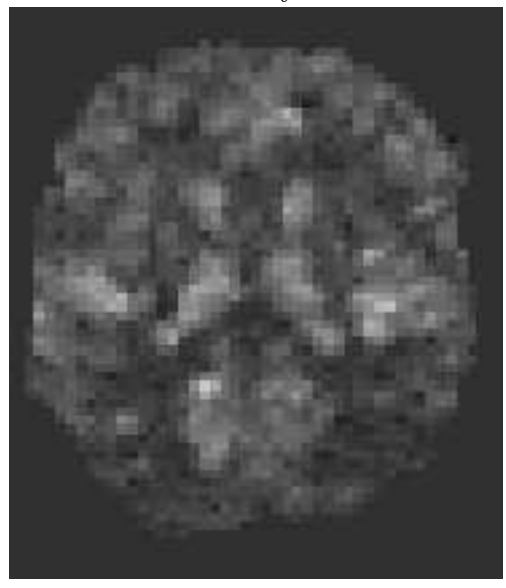

NL-time

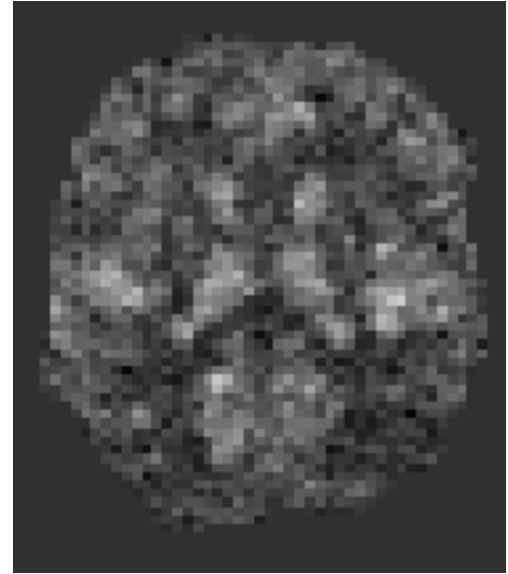

Gauss

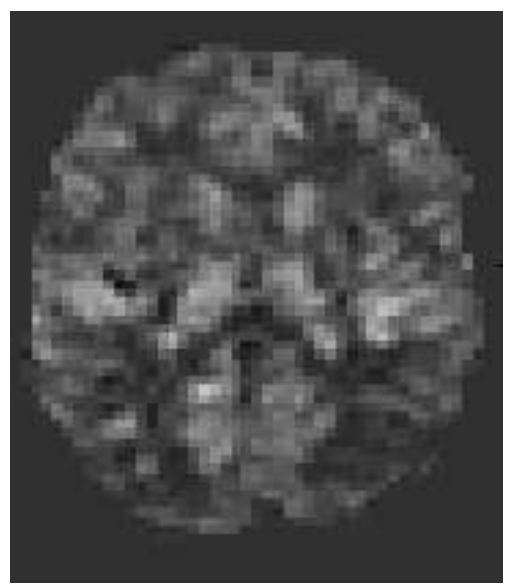

Ground truth

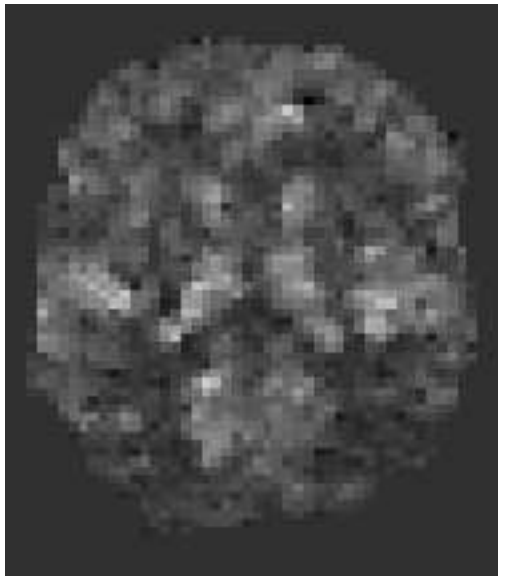

NL-space

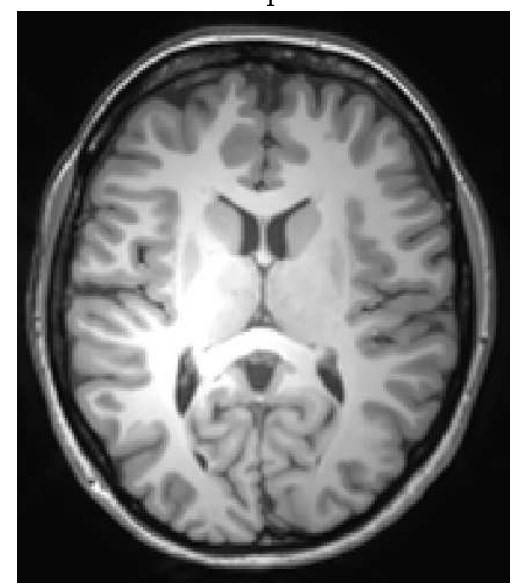

T1 image

Figure 5. Filtering of the dataset with added noise corresponding to 20 repetitions. The second slice is displayed for the input and ground-truth data and for the results of all the three filtering methods.

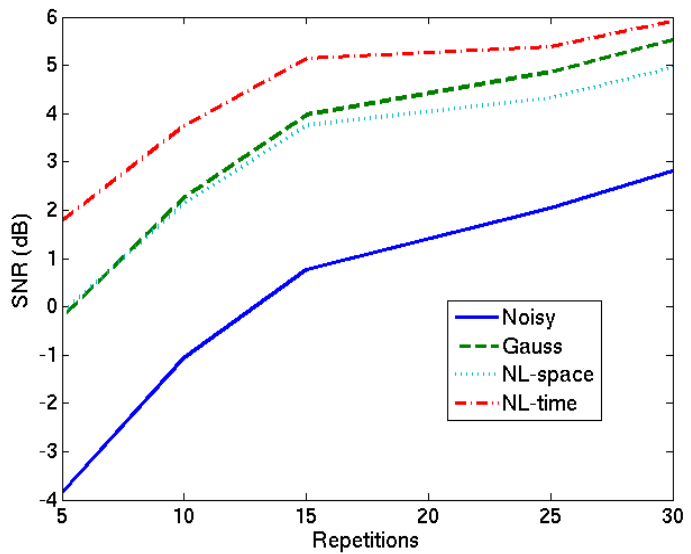

Figure 6. The SNR of the filtered data from 5,10,15,25 and 30 repetitions as compared with the average over 150 repetitions using leave-one-out cross validation. 


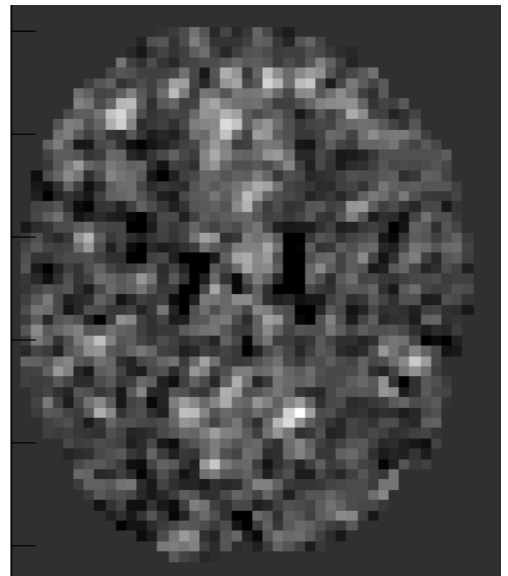

Noisy

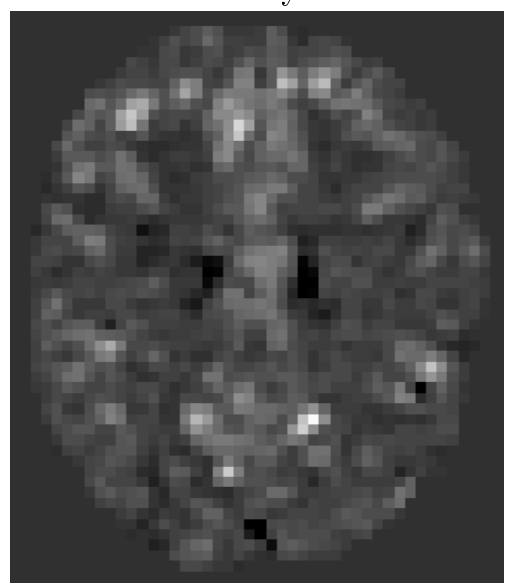

NL-time

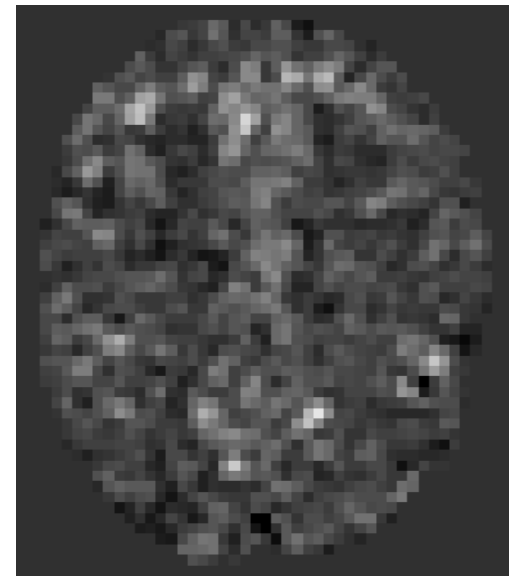

Gauss

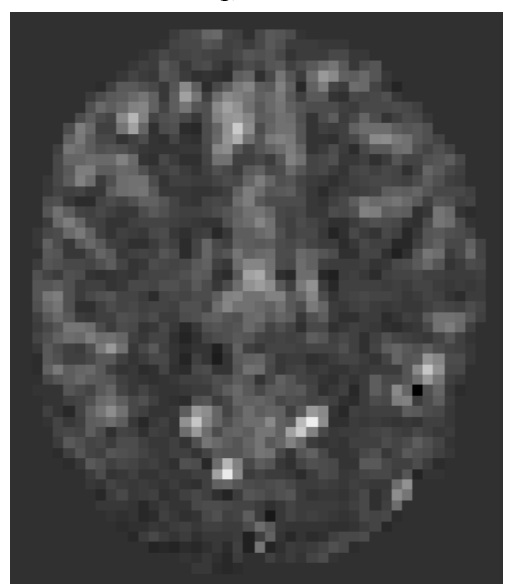

Ground truth

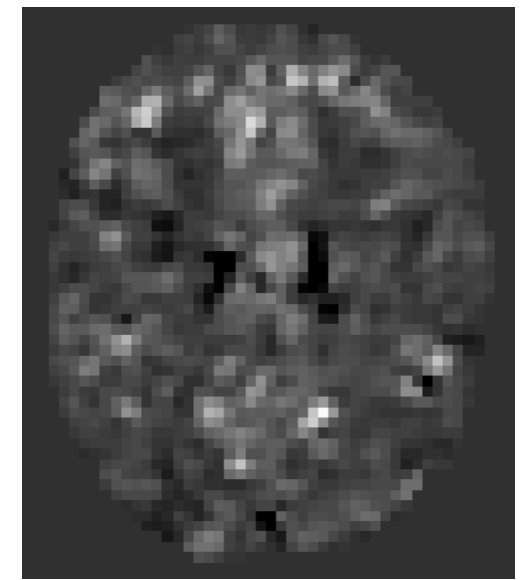

NL-space

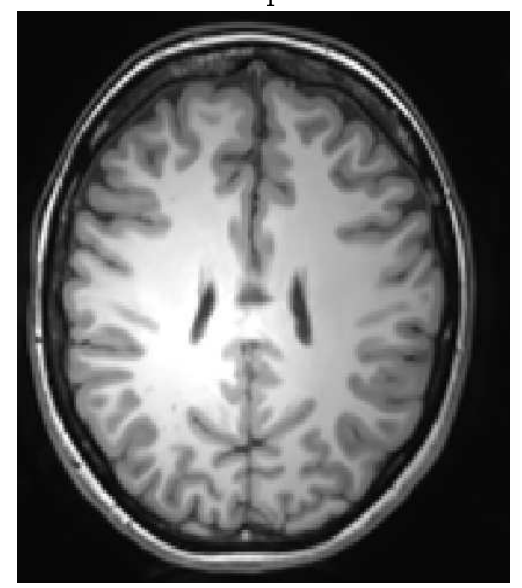

T1 image

Figure 7. Filtering of the real dataset of 20 repetitions. The fourth slice is displayed for the input and ground-truth data and the result of all the three filtering methods. 
was demonstrated in all tested cases. The method also produced better results in terms of SNR than Gaussian filtering in time which is, as far as we know, the only denoising methods used to filter multi-TI PASL sequences.

The automatic setting of the smoothing parameter $h^{12}$ of the time NL-means method based on noise variance provided sufficiently good results for all tested data. Equal weights were given to the time and the spatial neighborhood during the NL-means filtering with the extended neighborhood in the time domain. This weighting can be potentially changed to further increase the performance of the method.

The direct effect of the filtering on the CBF quantification also needs to be studied. In, ${ }^{5}$ Petersen et al. show that the increasing noise and subsequent regularization of the deconvolution can cause underestimation of the flow values. The noisy data also complicates quantification of flow by using an advanced perfusion models as several parameters usually needs to be estimated. ${ }^{2,8}$ The proposed method has the potential to reduce these problems.

\section{REFERENCES}

[1] Parkes, L. M. and Detre, J. A., [Quantitative MRI of the brain: Measuring changes caused by disease], ch. ASL: Blood perfusion measurements using arterial spin labelling, 455-473, John Wiley \& Sons (2003).

[2] Gallichan, D. and Jezzard, P., "Variation in the shape of pulsed arterial spin labeling kinetic curves across the healthy human brain and its implications for CBF quantification," Magnetic Resonance in Medicine 61(3), 686-695 (2009).

[3] Wong, E. C., Buxton, R. B., and Frank, L. R., "Quantitative imaging of perfusion using a single subtraction (QUIPSS and QUIPSS II)," Magnetic Resonance in Medicine 39, 702-708 (1998).

[4] Luh, W. M., Wong, E. C., Bandettini, P. A., and Hyde, J. S., "QUIPSS II with thin-slice TI1 periodic saturation: a method for improving accuracy of quantitative perfusion imaging using pulsed arterial spin labeling," Magnetic Resonance in Medicine 41, 1246-1254 (1999).

[5] Petersen, E., Lim, T., and Golay, X., "Model-free arterial spin labeling quantification approach for perfusion MRI," Magnetic Resonance in Medicine 55, 219-232 (2006).

[6] Gallichan, D. and Jezzard, P., "Modeling the effects of dispersion and pulsatility of blood flow in pulsed arterial spin labeling," Magnetic Resonance in Medicine 60(1), 53-60 (2008).

[7] Parkes, L. M., "Quantification of cerebral perfusion using arterial spin labeling: Two-compartement models," Journal of Magnetic Resonance Imaging 22, 732-736 (2005).

[8] Hrabe, J. and Lewis, D., "Two analytical solutions for a model of pulsed arterial spin labeling with randomized blood arrival times," Journal of Magnetic Resonance 167, 49-55 (March 2004).

[9] Wolf, R. L., Alsop, D. C., McGarvey, M. L., Maldjian, J. A., Wang, J., and Detre, J. A., "Susceptibility contrast and arterial spin labeled perfusion MRI in cerebrovascular disease," Journal of Neuroimaging 13(1), $17-27$ (2003).

[10] Wu, O., østergaard, L., Weisskoff, R. M., Benner, T., Rosen, B. R., and Sorensen, A. G., "Tracer arrival timing-insensitive technique for estimating flow in MR perfusion-weighted imaging using singular value decomposition with a block-circulant deconvolution matrix," Magnetic Resonance in Medicine 50(1), 164174 (2003).

[11] Buades, A., Coll, B., and Morel, J., "A review of image denoising algorithms, with a new one," Multiscale Modeling 63 Simulation 4(2), 490-530 (2005).

[12] Coupé, P., Yger, P., Prima, S., Hellier, P., Kervrann, C., and Barillot, C., "An optimized blockwise non local means denoising filter for 3D magnetic resonance images," IEEE Transactions on Medical Imaging 27, 425-441 (April 2008).

[13] Wiest-Daesslé, N., Prima, S., Coupé, P., Morrissey, S., and Barillot, C., "Non-local means variants for denoising of diffusion-weighted and diffusion tensor MRI," in [MICCAI'2007], 344-351 (2007).

[14] Wiest-Daesslé, N., Prima, S., Coupé, P., Morrissey, S., and Barillot, C., "Rician noise removal by non-local means filtering for low signal-to-noise ratio MRI: Applications to DT-MRI," in [MICCAI'2008], 171-179 (2008).

[15] Günther, M., Bock, M., and Schad, L. R., "Arterial spin labeling in combination with a look-locker sampling strategy: Inflow turbo-sampling epi-fair (its-fair)," Magnetic Resonance in Medicine 46, 974-984 (2001). 
[16] Ye, F. Q., Mattay, V. S., Jezzard, P., Frank, J. A., Weinberger, D. R., and McLaughlin, A. C., "Correction for vascular artifacts in cerebral blood flow values measured by using arterial spin tagging techniques," Magnetic Resonance in Medicine 37(2), 226-235 (1997).

[17] Lu, H., Clingman, C., Golay, X., and van Zijl, P. C. M., "Determining the longitudinal relaxation time (TI $\left.{ }_{1}\right)$ of blood at 3.0 Tesla," Magnetic Resonance in Medicine 52, 679-682 (2004).

[18] Wong, E. C., Buxton, R. B., and Frank, L. R., "Implementation of quantitative perfusion imaging techniques for functional brain mapping using pulsed arterial spin labeling," NMR in Biomedicine 10, 157-159 (1997). 